\title{
Computationally Tractable Model of Energy Detection Performance over Slow Fading Channels
}

\author{
Hongjian Sun, Student Member, IEEE, David I. Laurenson, Member, IEEE, \\ and Cheng-Xiang Wang, Senior Member, IEEE
}

\begin{abstract}
Energy detection (ED) has been widely used for detecting unknown deterministic signals in many wireless communication applications, e.g., cognitive radio, and ultra-wideband (UWB). However, the performance analysis of ED over slow fading channels is cumbersome, because it is difficult to derive closed-form expressions for the average probability of detection involving the generalised Marcum Q-function and the log-normal distribution. In this letter, we derive an approximation of the average probability of detection over a slow fading channel by replacing the log-normal distribution with a Wald distribution. In addition, we analyze the detection performance of the ED using a square-law combining scheme over multiple independent and identically distributed slow fading channels.
\end{abstract}

Index Terms-Energy detection, slow fading, cognitive radio, UWB, Wald distribution.

\section{INTRODUCTION}

$\mathbf{S}$ INCE Urkowitz's seminal paper [1], energy detection (ED, also known as radiometry) has been widely used for detecting unknown deterministic signals in many applications. For example, [2] employed ED for detecting the presence of ultra-wideband (UWB) signals, and [3] used ED to study the effect of the collaboration among cognitive radios. ED is commonly used not only due to its low computational and implementation complexity, but also because it does not need any prior knowledge of signals.

A non-fading additive white Gaussian noise (AWGN) channel is usually assumed when studying the performance of ED. However, in wireless communication applications, fading occurs because of multipath propagation and shadowing. The detection performance of ED over a variety of fading channels has gained interest recently in [4]-[6]. In [4], Digham et al. evaluated the performance of ED over Nakagami- $m$ fading channels and Rician fading channels. The influence of the slow fading on ED was numerically studied in [7], but without a closed-form expression. The challenge of deriving closedform expressions for the average probability of detection stems from the fact that it involves both the generalised Marcum Qfunction and the log-normal distribution.

Manuscript received June 1, 2010. The associate editor coordinating the review of this letter and approving it for publication was H.-H. Chen.

H. Sun and D. I. Laurenson are with the Institute for Digital Communication and with the Joint Research Institute for Signal and Image Processing, University of Edinburgh, Kings Buildings, Mayfield Road, Edinburgh, EH9 3JL, UK (e-mail: mrhjsun@hotmail.com, dave.laurenson@ed.ac.uk).

C.-X. Wang is with the Joint Research Institute for Signal and Image Processing, School of Engineering and Physical Sciences, Heriot-Watt University, Edinburgh, EH14 4AS, UK (e-mail: cheng-xiang.wang@hw.ac.uk).

The authors acknowledge the support of Wolfson Microelectronics Scholarship, and the Scottish Funding Council for the Joint Research Institute with the Heriot-Watt University which is a part of the Edinburgh Research Partnership.

Digital Object Identifier 10.1109/LCOMM.2010.090710.100934

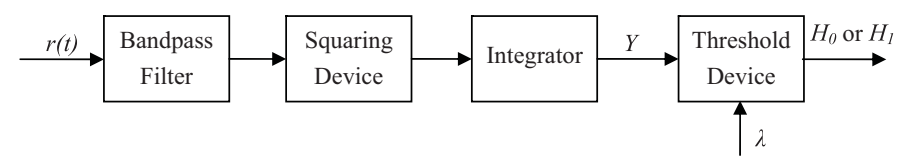

Fig. 1. Block diagram of the energy detector.

The contribution in this study is twofold. First, we give an approximation of the average probability of detection for the slow fading channel, by using the Wald distribution to replace the log-normal distribution. Second, we analyze the detection performance of the ED using a square-law combining (SLC) scheme over multiple independent and identically distributed (i.i.d.) slow fading channels, and derive a computationally tractable expression.

This paper is organized as follows. We briefly introduce the system model in Section II. In Section III, we derive an expression for the average probability of detection over a single slow fading channel. The detection performance of the ED using SLC over multiple slow fading channels is given in Section IV. Simulation results are presented in Section V, with conclusions given in Section VI.

\section{System Model}

A block diagram of an energy detector is shown in Fig. 1. The received signal, $r(t)$, is filtered by a bandpass filter (BPF), followed by a squaring device for measuring received energy, and an integrator that controls the observation interval, $T$. In order to decide whether the signal is present or not, the output of the integrator, $Y$, will act as a test statistic, and will be compared with a predetermined threshold, $\lambda$. The binary signal detection problem can be formulated as hypothesis test with $H_{0}$ (signal not present) or $H_{1}$ (signal present),

$$
\begin{array}{ll}
H_{0}: & r(t)=n(t), \\
H_{1}: & r(t)=h(t) s(t)+n(t),
\end{array}
$$

where $h(t)$ denotes the complex channel gain between the transmitter and the receiver, $s(t)$ denotes the bandlimited signal coming from the transmitter of unknown modulation format, and $n(t)$ is AWGN.

Following [1], the test statistic, $Y$, has the following distribution,

$$
Y \sim \begin{cases}\chi_{2 u}^{2}, & H_{0} \\ \chi_{2 u}^{2}(2 \gamma), & H_{1}\end{cases}
$$

where " " means "distributed as", $\gamma$ is the signal-to-noise ratio (SNR) at the receiver, and $\chi_{2 u}^{2}$ and $\chi_{2 u}^{2}(2 \gamma)$ denote the central and non-central chi-square distributions, respectively. Both distributions have the same degree of freedom (DoF), 
$2 u$ ( $u$ is the time bandwidth product), and the latter one has a non-central parameter $2 \gamma$.

The probability density function (PDF) of $Y$ is given as [4]

$$
f_{Y}(y)= \begin{cases}\frac{1}{2^{u} \cdot \Gamma(u)} \cdot y^{u-1} \cdot e^{-\frac{y}{2}}, & H_{0} \\ \frac{1}{2} \cdot\left(\frac{y}{2 \gamma}\right)^{\frac{u-1}{2}} \cdot e^{-\frac{2 \gamma+y}{2}} \cdot I_{u-1}(\sqrt{2 \gamma y}), & H_{1}\end{cases}
$$

where $\Gamma(a)$ is the gamma function and $I_{v}(a)$ is the $v^{t h}$ order modified Bessel function of the first kind.

For a non-fading AWGN channel, the probabilities of false alarm and detection are given in [4] as below

$$
\begin{aligned}
& P_{f a}=P_{r}\left(Y>\lambda \mid H_{0}\right)=\frac{\Gamma(u, \lambda / 2)}{\Gamma(u)}, \\
& P_{d}=P_{r}\left(Y>\lambda \mid H_{1}\right)=Q_{u}(\sqrt{2 \gamma}, \sqrt{\lambda}),
\end{aligned}
$$

where $\Gamma(a, x)$ denotes the incomplete gamma function given by $\Gamma(a, x)=\int_{x}^{\infty} t^{a-1} e^{-t} d t$, and $Q_{u}(a, x)$ denotes the generalised Marum Q-function given by

$$
Q_{u}(a, x)=\frac{1}{a^{u-1}} \int_{x}^{\infty} t^{u} e^{-\frac{a^{2}+t^{2}}{2}} I_{u-1}(a t) d t
$$

Another form of the generalised Marcum Q-function is given in (4.74) of [8] as

$$
Q_{u}(\sqrt{2 \gamma}, \sqrt{\lambda})=\sum_{n=0}^{\infty} e^{-\gamma} \frac{\gamma^{n}}{n !} \sum_{l=0}^{n+u-1} e^{-\frac{\lambda}{2}} \frac{\left(\frac{\lambda}{2}\right)^{l}}{l !} .
$$

With the aid of (8.352-2) in [9], we can rewrite above equation as

$$
Q_{u}(\sqrt{2 \gamma}, \sqrt{\lambda})=\sum_{n=0}^{\infty} \frac{\gamma^{n}}{n !} \frac{\Gamma\left(n+u, \frac{\lambda}{2}\right)}{\Gamma(n+u)} e^{-\gamma} .
$$

\section{Local Energy Detection in A Slow Fading CHANNEL}

When experiencing a fading channel, $P_{f a}$ in (4) will remain the same, since it is independent of the SNR. On the other hand, when the channel gain, $h(t)$, varies, the average probability of detection can be calculated by averaging $P_{d}$ in (5) over the SNR distribution as

$$
\overline{P_{d}}=\int_{0}^{\infty} P_{d}(\gamma, \lambda) f(\gamma) d \gamma=\int_{0}^{\infty} Q_{u}(\sqrt{2 \gamma}, \sqrt{\lambda}) f(\gamma) d \gamma
$$

where $f(\gamma)$ denotes the PDF of the SNR in a fading channel.

In terrestrial land-mobile wireless communication systems, the received SNR may be affected by the effect of shadowing due to objects obstructing the propagation path [8]. Empirical measurements showed that the received power fluctuates with a log-normal distribution about the area-mean power for various outdoor and indoor environments [7], [10]. The PDF of the SNR is given by [8]

$$
f(\gamma)=\frac{\xi}{\sqrt{2 \pi} \sigma \gamma} \exp \left(-\frac{\left(10 \log _{10} \gamma-\mu\right)^{2}}{2 \sigma^{2}}\right), \quad \gamma>0,
$$

where $\xi=10 / \ln (10), \mu(\mathrm{dB})$ denotes the area-mean SNR, and $\sigma(\mathrm{dB})$ denotes the standard deviation of $10 \log _{10} \gamma$. To the best of our knowledge, there exists no closed-form expression for the average probability of detection when we substitute (8) and (10) into (9). The log-normal distribution can be closely approximated by the Wald distribution (also known as the inverse Gaussian distribution) [11], [12], whose PDF is given by

$$
f(\gamma)=\sqrt{\frac{\eta}{2 \pi}} \gamma^{-3 / 2} \exp \left(-\frac{\eta(\gamma-\theta)^{2}}{2 \theta^{2} \gamma}\right), \quad \gamma>0,
$$

where $\theta=\mathrm{E}(\gamma)$ denotes the expectation of $\gamma$, and $\eta$ is the shape parameter. The variance of $\gamma$ is $\frac{\theta^{3}}{\eta}$, i.e., $\operatorname{Var}(\gamma)=\frac{\theta^{3}}{\eta}$. We propose to use the Wald distribution to approximate the log-normal distribution. In order to do so, by the method of moments we relate parameters $\eta, \theta$ with $\mu, \sigma$ as below

$$
\begin{aligned}
& \theta=\exp \left(\frac{\mu}{\xi}+\frac{\sigma^{2}}{2 \xi^{2}}\right), \\
& \eta=\frac{\theta}{\exp \left(\frac{\sigma^{2}}{\xi^{2}}\right)-1} .
\end{aligned}
$$

Substituting (8) and (11) into (9), with manipulation we obtain

$$
\begin{aligned}
\overline{P_{d, S h a}}= & \sqrt{\frac{\eta}{2 \pi}} e^{\frac{\eta}{\theta}} \sum_{n=0}^{\infty} \frac{\Gamma\left(n+u, \frac{\lambda}{2}\right)}{\Gamma(n+u) n !} \\
& \times \int_{0}^{\infty} \gamma^{n-\frac{3}{2}} e^{\left(-\frac{\eta / 2}{\gamma}-\frac{2 \theta^{2}+\eta}{2 \theta^{2}} \gamma\right)} d \gamma .
\end{aligned}
$$

Using (3.471-9) in [9] for calculating the integral, we obtain

$$
\begin{aligned}
\overline{P_{d, S h a}}= & \sqrt{\frac{2 \eta}{\pi}} e^{\frac{\eta}{\theta}} \sum_{n=0}^{\infty} \frac{\Gamma\left(n+u, \frac{\lambda}{2}\right)}{\Gamma(n+u) n !} \\
& \times\left(\sqrt{\frac{\eta \theta^{2}}{2 \theta^{2}+\eta}}\right)^{n-\frac{1}{2}} \mathrm{~K}_{n-\frac{1}{2}}\left(\frac{\sqrt{\eta\left(2 \theta^{2}+\eta\right)}}{\theta}\right),
\end{aligned}
$$

where $\mathrm{K}_{n-\frac{1}{2}}(a)$ denotes the modified Bessel function of the second kind with order $n-\frac{1}{2}$.

The truncation error, $T_{e}$, will be involved when using finite summations, $N$, to replace infinite summations in (14). Because $\frac{\Gamma(n, b)}{\Gamma(n)}=e^{-b} \sum_{i=0}^{n-1} \frac{b^{i}}{i !}$ can be viewed as the cumulative distribution function for a Poisson random variable of $X \sim \operatorname{Poi}(b)$, which results in $\frac{\Gamma(n, b)}{\Gamma(n)} \leq 1$, the truncation error is bounded by

$$
\begin{aligned}
T_{e} & \leq \sqrt{\frac{2 \eta}{\pi}} e^{\frac{\eta}{\theta}} \sum_{n=N+1}^{\infty} \frac{\left(\sqrt{\frac{\eta \theta^{2}}{2 \theta^{2}+\eta}}\right)^{n-\frac{1}{2}}}{n !} \mathrm{K}_{n-\frac{1}{2}}\left(\frac{\sqrt{\eta\left(2 \theta^{2}+\eta\right)}}{\theta}\right) \\
& =1-\sqrt{\frac{2 \eta}{\pi}} e^{\frac{\eta}{\theta}} \sum_{n=0}^{N} \frac{\left(\sqrt{\frac{\eta \theta^{2}}{2 \theta^{2}+\eta}}\right)^{n-\frac{1}{2}}}{n !} \mathrm{K}_{n-\frac{1}{2}}\left(\frac{\sqrt{\eta\left(2 \theta^{2}+\eta\right)}}{\theta}\right) .
\end{aligned}
$$

\section{Energy Detection over Slow Fading Channels}

The detection result of a single receiver may not be sufficiently reliable, which might be due to either the effect of fading or a low SNR. In such a scenario, diversity schemes are often employed because they can combat the severe fading [8]. Using SLC, the energy vectors, $Y_{1}, Y_{2}, \cdots, Y_{L}$, from $L$ distributed receivers are gathered at a fusion centre (FC), where the test statistic, $Y_{s l c}=\sum_{i=1}^{L} Y_{i}$ is formed [13]. When these $L$ fading channels are i.i.d., and all branches have the same noise variance, the fused energy, $Y_{s l c}$, has the following distribution,

$$
Y_{s l c} \sim \begin{cases}\chi_{2 L u}^{2}\left(2 \gamma_{s l c}\right), & H_{1} \\ \chi_{2 L u}^{2}, & H_{0},\end{cases}
$$


where $\gamma_{s l c}=\sum_{i=1}^{L} \gamma_{i}$ is given by [13].

In the case of non-fading AWGN channels, the probabilities of false alarm and detection under a SLC scheme can be given as below

$$
\begin{aligned}
P_{f a}^{\prime} & =\frac{\Gamma(L u, \lambda / 2)}{\Gamma(L u)}, \\
P_{d}^{\prime} & =Q_{L u}\left(\sqrt{2 \gamma_{s l c}}, \sqrt{\lambda}\right), \\
& =\sum_{n=0}^{\infty} \frac{\gamma_{s l c}^{n}}{n !} \frac{\Gamma\left(n+L u, \frac{\lambda}{2}\right)}{\Gamma(n+L u)} e^{-\gamma_{s l c}} .
\end{aligned}
$$

When the signal experiences fading over $L$ channels, the average probability of false alarm will remain the same as (17), and the average probability of detection can be evaluated by averaging $P_{d}^{\prime}$ over the SNR distribution as

$$
\begin{aligned}
\overline{P_{d}^{\prime}} & =\int_{0}^{\infty} P_{d}^{\prime}\left(\gamma_{s l c}, \lambda\right) f\left(\gamma_{s l c}\right) d \gamma_{s l c}, \\
& =\int_{0}^{\infty} Q_{L u}\left(\sqrt{2 \gamma_{s l c}}, \sqrt{\lambda}\right) f\left(\gamma_{s l c}\right) d \gamma_{s l c} .
\end{aligned}
$$

In slow fading channels, the PDF of the SNR in the node $i, \gamma_{i}$, can be approximated by a Wald distribution. When all fading channels are stationary and i.i.d., the condition $\frac{\eta_{i}}{\theta_{i}^{2}}=\frac{\mathrm{E}\left(\gamma_{i}\right)}{\operatorname{Var}\left(\gamma_{i}\right)}=b$ (constant) can be satisfied. Thus, the combined SNR under the SLC scheme, $\gamma_{s l c}$, will also follow the Wald distribution [14]. The PDF of $\gamma_{s l c}$ can be easily obtained by replacing each $\eta$ with $L \eta$, each $\theta$ with $L \theta$, and each $\gamma$ with $\gamma_{s l c}$ in (11). Using a similar method to that of the single slow fading channel, we can obtain the average probability of detection as below

$$
\begin{aligned}
\overline{P_{d, S h a}^{\prime}}= & \sqrt{\frac{2 L \eta}{\pi}} e^{\frac{\eta}{\theta}} \sum_{n=0}^{\infty} \frac{\Gamma\left(n+L u, \frac{\lambda}{2}\right)}{\Gamma(n+L u) n !} \\
& \times\left(\sqrt{\frac{\eta \theta^{2} L^{2}}{2 L \theta^{2}+\eta}}\right)^{n-\frac{1}{2}} \mathrm{~K}_{n-\frac{1}{2}}\left(\frac{\sqrt{\eta\left(2 L \theta^{2}+\eta\right)}}{\theta}\right) .
\end{aligned}
$$

The above result can also be obtained by replacing each $\eta$ with $L \eta$, each $\theta$ with $L \theta$, and each $u$ with $L u$ in (14).

\section{Simulation Results}

Receiver operating characteristic (ROC) analysis has been widely used in the signal detection theory. It is an ideal technique to quantify the tradeoff between the probability of detection and the probability of false alarm. In the simulation, we use complementary ROC curves $\left(P_{f a}\right.$ vs $\left.1-P_{d}\right)$ to show the detection performance of ED over slow fading channels. As we used the Wald distribution to approximate the lognormal distribution for deriving the average probability of detection, we compare the theoretical result in (14) with simulated result in Fig. 2. From both figures, we can find that the theoretical results closely fit the experimental results. In addition, we can see that, when the average probability of false alarm decreases, the approximation error slightly increases. This phenomenon may stem from the long right tail of the long-normal distribution, which is difficult to match. As shown in the right-hand figure, the mismatch becomes larger when the shadow standard deviation, $\sigma$, becomes larger (equivalent to a longer right tail in the log-normal distribution).
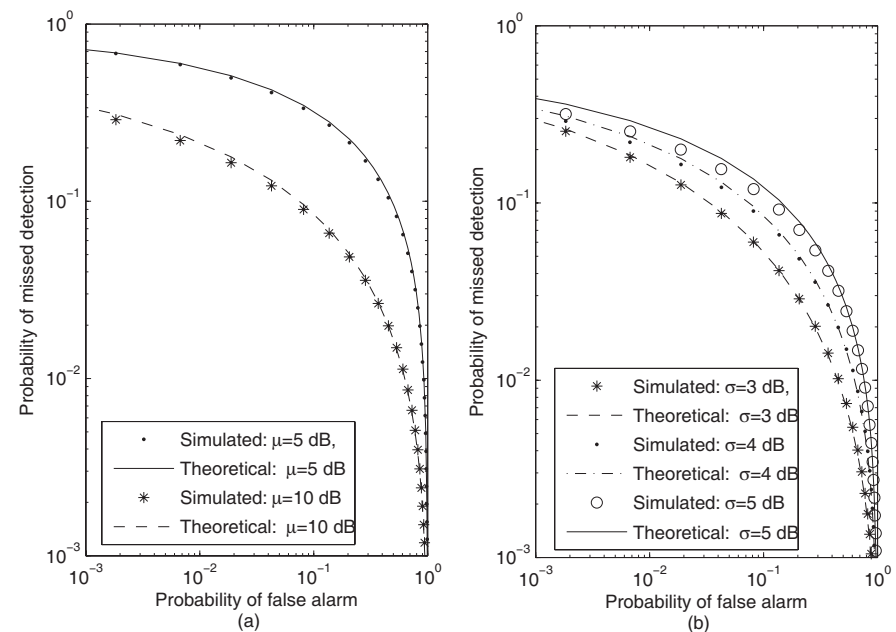

Fig. 2. Complementary ROC curves of energy detection over a slow fading channel with (a) the shadow standard deviation $\sigma=4 \mathrm{~dB}$, and (b) the areamean SNR $\mu=10 \mathrm{~dB}$, compared with theoretical result in (14).

\section{CONCLUSiOnS}

In this study, we have obtained a computationally tractable expression for the average probability of detection over a slow fading channel, by using the Wald distribution to replace the log-normal distribution. Using SLC, we have studied the detection performance of ED over i.i.d. slow fading channels. It has been shown that the theoretical expression closely match the experimental results. Since the effect of the long right tail in the log-normal distribution, the mismatch becomes larger when the shadow standard deviation increases.

\section{REFERENCES}

[1] H. Urkowitz, "Energy detection of unknown deterministic signals," Proc. IEEE, vol. 55, no. 4, pp. 523-531, Apr. 1967.

[2] C. Steiner and A. Wittneben, "Low complexity location fingerprinting with generalized UWB energy detection receivers," IEEE Trans. Signal Process., vol. 58, no. 3, pp. 1756-1767, Mar. 2010.

[3] C.-X. Wang, H.-H. Chen, X. Hong, and M. Guizani, "Cognitive radio network management: tuning in to real-time conditions," in IEEE Veh. Technol. Mag., vol. 3, no. 1, pp. 28-35, Mar. 2008.

[4] F. F. Digham, M.-S. Alouini, and M. K. Simon, "On the energy detection of unkown signals over fading channels," in Proc. IEEE ICC, May 2003, pp. 3575-3579.

[5] V. Kostylev, "Energy detection of a signal with random amplitude," in Proc. IEEE ICC, 2002, pp. 1606-1610.

[6] K. Ruttik, K. Koufos, and R. Jantti, "Detection of unknown signals in a fading environment," IEEE Commun. Lett., vol. 13, no. 7, pp. 498-500, July 2009.

[7] A. Ghasemi and E. Sousa, "Collaborative spectrum sensing for opportunistic access in fading environments," in Proc. IEEE DySPAN, Nov. 2005, pp. 131-136.

[8] M. K. Simon and M.-S. Alouini, Digital Communication over Fading Channels, 2nd edition. John Wiley \& Sons, Inc., 2004.

[9] I. S. Gradshteyn and I. M. Ryzhik, Table of Integrals, Series, and Products, 5th edition, A. Jeffrey, editor. Academic Press, Inc., 1994.

[10] H. Suzuki, "A statistical model for urban radio propagation," IEEE Trans. Commun., vol. 25, no. 7, pp. 673-680, July 1977.

[11] A. H. Marcus, "Power sum distributions: an easier approach using the Wald distribution," J. American Statistical Assoc., vol. 71, pp. 237-238, 1976.

[12] Karmeshu and R. Agrawal, "On efficacy of Rayleigh-inverse Gaussian distribution over K-distribution for wireless fading channels," Wireless Commun. and Mobile Computing, vol. 7, no. 1, pp. 1-7, 2007.

[13] F. F. Digham, M.-S. Alouini, and M. K. Simon, "On the energy detection of unknown signals over fading channels," IEEE Trans. Commun., vol. 55, no. 1, pp. 21-24, Jan. 2007.

[14] R. S. Chhikara and J. L. Folks, The Inverse Gaussian Distribution: Theory, Methodology, and Applications. Marcel Dekker Inc., 1989. 